\title{
BMJ Open Using kernel density estimation to understand the influence of neighbourhood destinations on BMI
}

\author{
Tania L King, ${ }^{1}$ Rebecca J Bentley, ${ }^{1}$ Lukar E Thornton, ${ }^{2}$ Anne M Kavanagh ${ }^{1}$
}

To cite: King TL, Bentley RJ, Thornton LE, et al. Using kernel density estimation to understand the influence of neighbourhood destinations on BMI. BMJ Open 2016;6: e008878. doi:10.1136/ bmjopen-2015-008878

- Prepublication history and additional material is available. To view please visit the journal (http://dx.doi.org/ 10.1136/bmjopen-2015008878).

Received 24 May 2015 Revised 16 January 2016 Accepted 18 January 2016

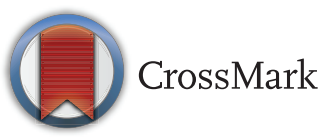

${ }^{1}$ Department of Gender and Women's Health, Centre for Health and Society, Academic Centre for Health Inequity, Melbourne School of Population Health, University of Melbourne, Melbourne, Victoria, Australia

${ }^{2}$ Centre for Physical Activity and Nutrition Research, School of Exercise and Nutrition Sciences, Deakin University, Burwood, Victoria, Australia

Correspondence to Dr Tania L King; tking@ unimelb.edu.au

\section{ABSTRACT}

Objectives: Little is known about how the distribution of destinations in the local neighbourhood is related to body mass index (BMI). Kernel density estimation (KDE) is a spatial analysis technique that accounts for the location of features relative to each other. Using $K D E$, this study investigated whether individuals living near destinations (shops and service facilities) that are more intensely distributed rather than dispersed, have lower BMls.

Study design and setting: A cross-sectional study of 2349 residents of 50 urban areas in metropolitan Melbourne, Australia.

Methods: Destinations were geocoded, and kernel density estimates of destination intensity were created using kernels of 400, 800 and $1200 \mathrm{~m}$. Using multilevel linear regression, the association between destination intensity (classified in quintiles Q1 (least)-Q5(most)) and BMI was estimated in models that adjusted for the following confounders: age, sex, country of birth, education, dominant household occupation, household type, disability/injury and area disadvantage. Separate models included a physical activity variable.

Results: For kernels of 800 and $1200 \mathrm{~m}$, there was an inverse relationship between $\mathrm{BMI}$ and more intensely distributed destinations (compared to areas with least destination intensity). Effects were significant at $1200 \mathrm{~m}: \mathrm{Q} 4, \beta-0.86,95 \% \mathrm{Cl}-1.58$ to -0.13 , $\mathrm{p}=0.022 ; \mathrm{Q}, \beta-1.0395 \% \mathrm{Cl}-1.65$ to -0.41 , $\mathrm{p}=0.001$. Inclusion of physical activity in the models attenuated effects, although effects remained marginally significant for Q5 at $1200 \mathrm{~m}: \beta-0.7795 \% \mathrm{Cl}-1.52$, $-0.02, p=0.045$.

Conclusions: This study conducted within urban Melbourne, Australia, found that participants living in areas of greater destination intensity within $1200 \mathrm{~m}$ of home had lower BMls. Effects were partly explained by physical activity. The results suggest that increasing the intensity of destination distribution could reduce BMI levels by encouraging higher levels of physical activity.

\section{INTRODUCTION}

Obesity remains a growing problem in many Western countries including Australia, where $63 \%$ of the adult population is overweight or obese. ${ }^{1}$ Among developed countries, the
Strengths and limitations of this study

- Kernel density estimation represents advancement in the study of the relationship between the built environment and body mass index (BMI).

- Exposure areas were specific to individual respondents.

- The use of multiple kernel distances enables the comparison of distance effects

- There may be measurement error associated with self-reported physical activity and BMI.

- There is some potential misclassification and systematic error associated with BMI and physical activity.

economic costs associated with overweight and obesity are significant. ${ }^{2}$ There is growing interest in understanding how the neighbourhood environment may influence the risk of overweight and obesity by encouraging increased energy consumption and discouraging energy expenditure. While it seems plausible that the rise in obesity can be partly attributed to the built environment, the abundant literature examining aspects of the built environment in relation to weight status has yielded equivocal results, with calls for better metrics to evaluate associations. ${ }^{3}$ Examination of destinations, an increasingly common focus of neighbourhood research, has yielded mixed results: inverse relationships between body mass index (BMI) and grocery or supermarket store availability have been observed in some research, ${ }^{4-6}$ while positive relationships have been noted elsewhere between BMI and destinations such as small food stores and supermarkets, ${ }^{7}$ and fast-food stores. ${ }^{4}$

The limitations of standard approaches in operationalising elements of the built environment may explain some of the contradictory findings. Most commonly, access to destinations in neighbourhoods has been measured in terms of the destinations present within a defined catchment or buffer (ie, a count of the number of destinations within a certain distance of home, or the presence of 
destinations within a defined area). Such measures have been criticised on the basis of their binary or categorical classification: a feature (in this case destination) is simply classified as present or absent. ${ }^{9}{ }^{10}$ A destination located at the edge of the areal unit is not equivalent to a more proximal destination, however, typical binary measures do not accommodate this, and analyse them as if their effect is the same. Furthermore, such measures of destination accessibility do not take into account the location of destinations relative to each other (ie, they provide no indication of whether they are intensely distributed or dispersed).

Kernel density estimation (KDE) - a spatial analysis technique that accounts for the location of features (ie, destinations) relative to each other-is an emerging spatial tool that has previously been applied to the examination of various aspects of the environment, such as park access, ${ }^{10}$ health resources, ${ }^{11}$ and recently, the food environment. ${ }^{1213}$ The ability to weight the distribution of destinations according to their proximity to a central feature or location is one of the key imperatives for the use of KDE. Further, by representing the distribution of activity or exposures on a continuous surface, KDE helps identify the presence of clusters and irregularities. ${ }^{14}$ In plain terms, the use of KDE to examine the distribution of destinations in neighbourhoods enables researchers to see where destinations are sparsely distributed (dispersed), and where they are more intensely distributed (clustered). There is a paucity of studies that have used KDE to examine the relationship between destinations and BMI. In the USA, researchers have applied KDE methods to the examination of the relationship between the intensity/density of elements of the built environment and BMI, or obesity. ${ }^{13}{ }^{15}$ Among a sample of adults with diabetes, one study created a food environment score by subtracting the kernel density estimate of unhealthy food stores from that of healthy food stores, and examined associations between the food environment score and obesity. ${ }^{15}$ They found that for higher income groups, a healthier food environment was associated with lower rates of obesity, but for lower income groups, higher rates of obesity were observed among those living in a healthier food environment. ${ }^{15}$ These findings led them to conclude that the food environment can have differential effects on residents depending on the pressures placed on individual financial resources. ${ }^{15}$ Also in the USA, food stores were classified as either BMI-healthy, BMI-intermediate, or BMI-unhealthy, and kernel density estimates of the distribution of each of these was examined in relation to BMI. ${ }^{13}$ Greater density of BMI-healthy stores was found to be significantly associated with reduced BMI. ${ }^{13}$ In reviewing the literature, we did not encounter any research examining the relationship between BMI and kernel density estimates of destination intensity in Australia. Furthermore, previous studies using kernel density estimates of destinations in relation to BMI have only examined food purchasing destinations. ${ }^{13}{ }^{15} \mathrm{We}$ contend that it is important to consider other neighbourhood destinations when examining the relationship between neighbourhood environments and BMI. Certainly the motivations for examining BMI in relation to food and recreational destinations are sound and logical: the intensity of healthy or unhealthy food stores may lead to greater consumption of healthy or unhealthy foods, and impact on BMI; the presence of recreational facilities could encourage greater levels of physical activity and lead to reduced BMI levels. However, we argue that many other destinations may also influence BMI by making active travel a viable option. The importance of other destinations is supported by our previous work on this data set. We have previously found strong associations between several specific destination types, such as community resources and small food stores, and both walking and physical activity. ${ }^{16}$ Furthermore, in other analyses we used KDE to show that residents of areas with greater destination intensity (as measured by KDE) walked more frequently, and showed higher odds of being sufficiently active (this being largely explained by levels of walking). ${ }^{17}$

The impetus for broadening the focus to other destinations therefore, is thus: greater intensity of many destinations (not just food and recreational destinations) may present residents with more opportunities for active travel, and thereby encourage incidental physical activity. By encouraging more walking, cycling and physical activity, it is plausible that greater density of a range of destinations may be associated with lower BMI levels.

In this paper, we test whether the intensity of destinations near participants' homes is related to reduced BMIs, and assess whether this relationship is mediated by residents' overall physical activity levels. Based on our previous findings that an increased level of KDE is associated with higher levels of walking and greater odds of being sufficiently active ${ }^{17}$ we hypothesised that increasing levels of destination intensity, as measured by $\mathrm{KDE}$, would be inversely associated with BMI, and that this would be mediated by physical activity.

\section{METHODS}

The analyses are based on individual and area-level data collected as part of the Victorian Lifestyle and Neighbourhood Environment Study (VicLANES) from 2349 individuals in 50 small areas in metropolitan Melbourne. Additional information on areas was also obtained from a range of different administrative geospatial data sets. The VicLANES project design was approved by the La Trobe University Human Ethics Committee (\#02-130), and free and informed consent was obtained from all participants.

\section{Study setting and design}

VicLANES was a large, multilevel study conducted in 2003-2004 across the 21 innermost local government areas in Melbourne, Australia. The VicLANES methods 
have been reported previously. ${ }^{18} 19$ Briefly, CCDs (otherwise known as census collection districts; at the time of the study these were the smallest geographic unit of measurement used by the Australian Bureau of Statisics) were ranked according to a household measure of low income $(\angle \$ A 400 /$ week $)$, then stratified into septiles. Fifty CCDs were then randomly selected from the top (17), middle (16) and bottom (17) septile. Postal questionnaires were sent to 4005 residents over the age of 18 years, who were randomly selected from the electoral roll (voting is compulsory for all Australians over the age of 18 years, and it is estimated that $97.7 \%$ of those eligible to vote are enrolled do so). ${ }^{20}$ The Tailored Design Method for Mail Surveys ${ }^{21}$ was adopted to maximise response rates. A $58.7 \%$ valid completion rate was achieved, with 2349 participants returning a valid survey about their physical activity behaviour. Respondents were aged $18-75$ years.

\section{Outcome measure}

The outcome variable, BMI, was based on self-reported height and weight and was modelled as a continuous outcome.

\section{Exposure variable: destinations}

Destination information came from two principal sources: (1) the VicLANES environmental audit, ${ }^{22}{ }^{23}$ and (2) publicly available spatial data sets. We chose destinations that we thought people may use active travel to access in their neighbourhood. Destinations included in the analysis were: educational facilities (schools, kindergartens, universities), café/takeaway stores, transport stops and stations, supermarkets, sports facilities, community resources (such as libraries, maternal and child health centres, places of worship, community centres), small food stores (such as convenience stores, bakeries, butchers, green grocers). Online supplementary table S1 provides details of the destination types and the sources of this destination data.

\section{KDE: constructing the exposure variable in ArcGIS}

In ArcGIS 10.1, ${ }^{24}$ all destinations were combined and merged into a single layer. The kernel density surface of destinations was estimated and extracted using the 'extract values to points' command in the Spatial Analyst toolbox in ArcGIS. ${ }^{24}$

The process of $\mathrm{KDE}$ commences with a continuous map surface divided into a grid of specified cell sizes. Across this map, KDE fits a series of cones or kernels centred over each point feature of interest (in this case destinations), creating a continuous map of feature density or intensity. ${ }^{25}$ The radius of each cone/kernel is set to a distance that is estimated to reflect the service area/area of effect of that particular feature or resource. Each cell on the map surface is assigned a kernel density estimate such that cells at the centre of the cone receive higher estimates, and cells at the cone's periphery receive smaller estimates. ${ }^{25}$ In effect, kernel density estimates are inversely related to the distance from the feature that the cone is centred on (the centre of the cone).$^{25} \mathrm{KDE}$ weights the effect of features such that a feature located closest to a point/location of interest is assigned greater weighting, while a feature located some distance away receives a negligible weighting. ${ }^{14}$ The cones of different features/destinations overlap, often substantially. A smoothing function (bivariate Gaussian distribution) adds the estimates of overlapping kernels for each cell. ${ }^{12}{ }^{25}$ An example of the resultant image of KDE of the distribution of destinations using $1200 \mathrm{~m}$ kernels is presented in figure 1.

The kernel density values were extracted so that each participant's household location was assigned the kernel density value of the output cell in which they resided. While kernel density estimates are calculated on the basis of how close the destinations are to each other, the values extracted at each participant location provide an indication of the proximity and density of destinations in relation to the participant location. High kernel density estimates indicate high intensity or clustering of destinations, low kernel density estimates indicate negligible, highly dispersed destinations. Moderate kernel density estimates may indicate dispersed destinations, or they may result when a participant is located a greater distance from a set of highly clustered destinations.

In this analysis, kernel density estimates were calculated using kernel sizes of 400, 800 and $1200 \mathrm{~m}$. We were interested in the extent to which physical activity might mediate any observed relationships between destinations and BMI. It is argued that $400 \mathrm{~m}$ is the distance that people may choose to walk rather than drive, ${ }^{26}$ and this approximately equates to a 5 minute walk. Distances of 800 and $1200 \mathrm{~m}$ were also chosen, as they represent the distance that the average person could walk in 10 and $15 \mathrm{~min}$, respectively.

Constructing the exposure variable for statistical analysis Kernel density estimates were categorised into quintiles (quintile 1 representing areas of least intensely

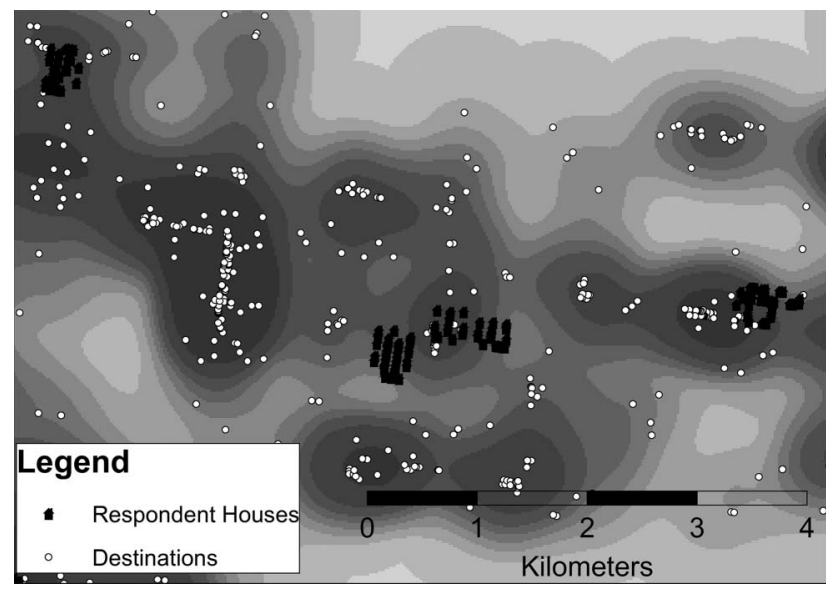

Figure 1 Raster representation of kernel density estimates of destination distribution using $1200 \mathrm{~m}$ kernels. 
distributed destinations, and quintile 5 representing areas of most intensely distributed destinations). There are precedents for the use of quintiles to model the distribution of destinations, including research in the $\mathrm{USA}^{13}$ and our own research. ${ }^{16}$

\section{Confounders}

Based on the literature, several covariates were included in the analysis as potential confounders. ${ }^{13}{ }^{27}$ These were: age (grouped into six categories: 18-24; 25-34; 35-44; 45-54; 55-64; 64 years and over), sex, country of birth (born in Australia; born in a country other than Australia), education (bachelor degree or higher; diploma; vocational training; and no postschool qualification), household type (single adult-no children; single adult with children; two or more adults-no children; two or more adults with children), dominant household occupation (professional; white-collar employee; bluecollar employee; not in labour force-including retirees, students, unemployed, those not looking for, or unable to work), and disability/injury that prevents exercise (yes, no). Area disadvantage was also included as a potential confounder. The three septiles used to set the sample frame (see 'Study settings and design' above) were used as an indicator of area disadvantage, and were defined as least disadvantaged, mid-disadvantaged and most disadvantaged.

\section{Physical activity sufficiency}

Using items from the Active Australia Survey, participants were asked to indicate the frequency and duration of their participation in walking, vigorous physical activity, moderate physical activity, vigorous garden or yard work. These items were then used to produce a measure of overall physical activity sufficiency. The Active Australia Survey has been used in national surveys, and demonstrates very good reliability and validity. ${ }^{28}$

Australian and international guidelines recommend that a person needs to participate in at least $30 \mathrm{~min}$ of moderate to vigorous intensity activity most days of the week, for a total of at least $150 \mathrm{~min}$ of activity per week. ${ }^{28-}$ 30 According to the Active Australia Survey guidelines, physical activity sufficiency for health can be measured in two ways ${ }^{28}$ : (1) measured as total time engaged in physical activity (at least $150 \mathrm{~min}$ for sufficiency); (2) measured as total time across total number of sessions (at least $150 \mathrm{~min}$ across at least five sessions). The combined measure of time and number of sessions (at least $150 \mathrm{~min}$ of at least moderate intensity activity across at least five session week) ${ }^{31} 32$ was chosen for this analysis, because it most closely matches guidelines for physical activity sufficiency. ${ }^{29}$

In accordance with the Active Australia Survey administration and implementation guidelines, VicLANES responses were converted to total amount of time (minutes) engaged in each activity, and summed, with vigorous activity weighted by a factor of $2 . .^{28} 33$ Participants were then categorised into one of two groups: those reporting less than $150 \mathrm{~min}$ of at least moderate activity across five sessions a week were classified as insufficiently active; those with at least $150 \mathrm{~min}$ of at least moderate activity across five sessions or more were classified as sufficiently active.

\section{Statistical analysis}

Pregnant women $(n=22)$ were excluded because their BMI may have been altered by their pregnancy status. One CCD from just outside the central business district of Melbourne was omitted from the final analysis $(n=14)$ as this CCD's catchment area encapsulated almost the entire central business district, and the number of features and destinations contained in this catchment area was irregularly high. We also excluded 150 participants for whom BMI data were missing, resulting in an analytical sample of 2163 participants, and 49 CCDs. There was no missing data for sex, age group or level of area disadvantage. Missing data for the other variables ranged from $0.1 \%$ to $2.1 \%$, with the exception of the disability item and the physical activity item, for which missing data amounted to $5.6 \%$ and $14.2 \%$, respectively.

All analyses were conducted in Stata IC 10.0. The referent category for the exposure was quintile 1 (Q1, lowest destination clustering). Descriptive analyses included cross-tabulations between BMI and both individual covariates and kernel density estimates. Multilevel linear regression was performed (with CCDs at level 2 and individuals at level 1) to examine the associations between BMI and the three kernel density measures $(400,800$ and $1200 \mathrm{~m}$ ). More specifically, we used mixed-effects multilevel models with robust SEs. All models were adjusted for confounders. Finally, physical activity was included in the models to test whether it attenuated associations between kernel density estimates and BMI. ORs and 95\% CIs are reported for all estimates.

\section{RESULTS}

\section{Descriptive statistics}

Summary statistics for the outcome by different covariates are presented in table 1. Higher BMIs were reported among men, those aged 55-64 years and over 65 years, those living in the most disadvantaged areas, and those missing data for education (while the BMI for those missing information on country of birth was high, this group only constituted two participants). Lower BMIs were reported among women, those with a bachelor degree or higher, those in the least disadvantaged areas, and younger participants (aged 18-24 and 2534 years).

\section{Multilevel analysis: KDE}

Table 2 shows the adjusted results of the multilevel analyses that tested the association between BMI and the kernel density estimates for destination intensity at kernel sizes of 400,800 and $1200 \mathrm{~m}$. There was no 
Table 1 Sample descriptive statistics (unweighted)

\begin{tabular}{lll}
$\substack{\text { Sample } \\
\mathrm{n}=2163}$ & BMI \\
\cline { 1 - 1 } $\mathrm{n} \quad \%$ & & \\
& &
\end{tabular}

Sex

Male

Female

Missing

Country of birth

Australia

Elsewhere

Missing

Age (years)

18-24

25-34

$35-44$

$45-54$

$55-64$

Over 65

Missing

Dominant occupation (household)

Professionals

White collar

Blue collar

Not in labour force

Missing

Education

Bachelor degree or higher $\quad \begin{array}{llll}695 & 32.1 & 24.8(4.1)\end{array}$

Diploma

Vocational

No postschool qualifications

Missing

Household type

Single adult, no children

Single adult, children

Two or more adults, no

children

Two or more adults, children

Missing

Level of area disadvantage

Least disadvantaged

Mid-disadvantaged

Most disadvantaged

Missing

Disability or injury

Yes

No

Missing

Physical activity sufficiency

Insufficiently active

Sufficiently active

Missing

BMI, body mass index.

\begin{tabular}{rcl}
961 & 44.4 & $26.1(3.8)$ \\
1202 & 55.6 & $25.0(4.9)$ \\
0 & 0 & n/a \\
& & \\
1531 & 70.8 & $25.5(4.5)$ \\
630 & 29.1 & $25.6(4.3)$ \\
2 & 0.1 & $31.6(1.8)$ \\
& & \\
170 & 7.9 & $23.5(4.6)$ \\
374 & 17.3 & $24.8(4.5)$ \\
468 & 21.6 & $25.6(4.3)$ \\
459 & 21.2 & $25.9(4.6)$ \\
369 & 17.1 & $26.0(4.0)$ \\
323 & 14.9 & $26.3(4.5)$ \\
0 & 0 & $n / a$ \\
& & \\
1016 & 47.0 & $25.3(4.1)$ \\
327 & 15.1 & $25.7(5.1)$ \\
224 & 10.4 & $25.5(4.1)$ \\
551 & 25.5 & $25.8(4.7)$ \\
45 & 2.1 & $25.9(6.2)$ \\
& & \\
695 & 32.1 & $24.8(4.1)$ \\
242 & 11.2 & $26.0(4.5)$ \\
405 & 18.7 & $26.0(4.4)$ \\
776 & 35.9 & $25.7(4.7)$ \\
45 & 2.1 & $27.0(5.3)$ \\
& & \\
370 & 17.1 & $25.8(4.8)$ \\
125 & 5.8 & $26.0(5.0)$ \\
898 & 41.5 & $25.2(4.3)$ \\
& & \\
730 & 33.8 & $25.7(4.3)$ \\
40 & 1.9 & $25.8(5.4)$ \\
& & \\
789 & 36.5 & $25.0(3.9)$ \\
725 & 33.5 & $25.5(4.6)$ \\
649 & 30.0 & $26.1(4.8)$ \\
0 & 0 & $n$ \\
& & \\
457 & 21.1 & $26.7(5.0)$ \\
1578 & 73.0 & $25.2(4.3)$ \\
128 & 5.9 & $25.4(4.2)$ \\
& & \\
1011 & 46.7 & $25.8(4.7)$ \\
845 & 39.1 & $25.1(3.9)$ \\
307 & 14.2 & $25.6(4.9)$ \\
\hline & &
\end{tabular}

\begin{tabular}{llll}
307 & 14.2 & 25.6 & $(4.9)$ \\
\hline
\end{tabular}

association between kernel density estimates and BMI for the $400 \mathrm{~m}$ kernel size. For both the 800 and $1200 \mathrm{~m}$ kernels, however, increasing kernel density estimates were associated with a reduced BMI, with significant results observed at $1200 \mathrm{~m}$. Evidence was strongest for quintiles 4 and 5 relative to quintile 1 at $1200 \mathrm{~m}$

(quintile $4,-0.86 \mathrm{~kg} / \mathrm{m}^{2}$, quintile $5,-1.03 \mathrm{~kg} / \mathrm{m}^{2}$ ). Inclusion of physical activity attenuated these effects (quintile $4,-0.75 \mathrm{~kg} / \mathrm{m}^{2}$; quintile $5,-0.77 \mathrm{~kg} / \mathrm{m}^{2}$ ).

\section{Sensitivity analysis}

Results remained substantively unchanged in models that excluded transport destinations. We also ran models in which kernel density estimates were modelled as continuous variables, rather than categorical. These results supported those presented in table 2, with significant effects observed for $1200 \mathrm{~m}$, but not other distances.

\section{DISCUSSION}

In the analysis presented in this paper, the intensity of destinations was associated with BMI at $1200 \mathrm{~m}$ kernels. Specifically, as the intensity of destinations increased, the BMI of participants decreased, this being significant at $1200 \mathrm{~m}$ for quintiles 4 and 5 (the quintiles with the most intensely distributed destinations). This effect was attenuated with the inclusion of physical activity in the multilevel regression models.

According to these results for BMI (unadjusted for physical activity), using $1200 \mathrm{~m}$ kernels, the BMI of a $65 \mathrm{~kg}$ woman of $1.65 \mathrm{~m}$ height $(\mathrm{BMI}=23.9)$, living in an area of greatest destination intensity (quintile 5), would be $1.03 \mathrm{~kg} / \mathrm{m}^{2}$ less than if she was living in an area of least destination intensity (quintile 1) - or $2.9 \mathrm{~kg}$ lighter. A male of 1.75 metres in height, and $75 \mathrm{~kg}$ (with a BMI of 24.5) living in the quintile of most destination intensity compared to least destination intensity, would be $3.2 \mathrm{~kg}$ lighter. As we have previously pointed out, ${ }^{18}$ while such a shift in individual weight may not have a substantial impact on individual health and mortality, it may reduce the overall burden of obesity-related disease at a population level. ${ }^{34}$

The observed association between destination distribution and BMI at $1200 \mathrm{~m}$ is consistent with our hypothesis that more intensely distributed destinations would be associated with reduced BMI. We presupposed that any relationship between destination intensity and BMI would operate through increased physical activity: more destinations would increase residents' opportunities for physical activity (principally through active travel), and lead to reduced BMIs. Supporting this, the inclusion of physical activity sufficiency in the analytical models attenuated findings. These results are broadly consistent with several other studies revealing inverse associations between BMI and destinations, such as grocery stores or supermarkets, ${ }^{4-613}$ and bus and transit stops. ${ }^{35}$ It is difficult to place these results within the literature given there are scant studies examining associations between kernel density estimates of destination density and BMI. Both the studies that we are aware of that have examined the association between KDE and BMI distinguished between healthy and unhealthy food destinations. ${ }^{13} 15$ Rundle $e t a l^{13}$ found that the KDE of healthy destinations was inversely associated with BMI. In The Diabetes 
Table 2 Multilevel linear regression: $\beta$ coefficients for association between destination intensity and BMI

\begin{tabular}{|c|c|c|c|}
\hline \multirow[b]{2}{*}{ Kernel distance } & \multirow[b]{2}{*}{$\begin{array}{l}\text { Quintile of kernel density estimates } \\
\text { of destination intensity }\end{array}$} & \multicolumn{2}{|c|}{ Beta coefficient for change in BMI } \\
\hline & & $\begin{array}{l}\text { Model 1† } \\
\mathrm{n}=1927 \\
\mathrm{Resp} / \mathrm{CCD} \S=39.3\end{array}$ & $\begin{array}{l}\text { Model } 2 \ddagger \\
\mathrm{n}=1675 \\
\text { Resp/CCD } \$=34.2\end{array}$ \\
\hline \multirow[t]{7}{*}{$400 \mathrm{~m}$} & Quintile 1 & Referent & Referent \\
\hline & Quintile 2 & $-0.23(-0.76$ to 0.30$)$ & $-0.04(-0.59$ to 0.50$)$ \\
\hline & Quintile 3 & $0.13(-0.56$ to 0.82$)$ & $0.21(-0.58$ to 1.00$)$ \\
\hline & Quintile 4 & $-0.07(-0.69$ to 0.82$)$ & $-0.19(-0.61$ to 0.99$)$ \\
\hline & Quintile 5 & $0.18(-0.51$ to 0.87$)$ & $0.22(-0.53$ to 0.96$)$ \\
\hline & Area-level variance & 0.237 & 0.275 \\
\hline & ICC & $1.34 \%$ & $1.64 \%$ \\
\hline \multirow[t]{7}{*}{$800 \mathrm{~m}$} & Quintile 1 & Referent & Referent \\
\hline & Quintile 2 & $-0.38(-0.92$ to 0.16$)$ & $-0.41(-1.03$ to 0.22$)$ \\
\hline & Quintile 3 & $-0.31(-0.95$ to 0.33$)$ & $-0.30(-1.03$ to 0.43$)$ \\
\hline & Quintile 4 & $-0.63(-1.42$ to 0.16$)$ & $-0.48(-1.38$ to 0.42$)$ \\
\hline & Quintile 5 & $-0.61(-1.41$ to 0.18$)$ & $-0.32(-1.22$ to 0.58$)$ \\
\hline & Area-level variance & 0.164 & 0.232 \\
\hline & ICC & $0.93 \%$ & $1.39 \%$ \\
\hline \multirow[t]{7}{*}{$1200 \mathrm{~m}$} & Quintile 1 & Referent & Referent \\
\hline & Quintile 2 & $-0.42(-0.92$ to 0.10$)$ & $-0.53(-1.11$ to 0.05$)$ \\
\hline & Quintile 3 & $-0.42(-1.03$ to 0.19$)$ & $-0.48(-1.19$ to 0.24$)$ \\
\hline & Quintile 4 & $-0.86(-1.58 \text { to }-0.13)^{*}$ & $-0.75(-1.59$ to 0.09$)$ \\
\hline & Quintile 5 & $-1.03(-1.65 \text { to }-0.41)^{\star *}$ & $-0.77(-1.52 \text { to }-0.17)^{\star}$ \\
\hline & Area-level variance & 0.100 & 0.189 \\
\hline & ICC & $0.57 \%$ & $1.13 \%$ \\
\hline \multicolumn{4}{|c|}{$\begin{array}{l}\text { Estimates adjusted for: age, sex, country of birth, education, household, dominant household occupation, level of area disadvantage, injury/ } \\
\text { disability. } \\
{ }^{*} p<0.05 \text {. }\end{array}$} \\
\hline
\end{tabular}

Study of Northern California (DISTANCE), however, the relationship varied by income bracket and race: for all ethnic groups in the high-income bracket, greater density (KDE) of healthy food destinations was associated with reduced odds of being overweight or obese; while for those in the lower income category, having greater intensity of healthy food destinations (as measured by $\mathrm{KDE}$ ) was associated with greater odds of being overweight or obese, although this was only statistically significant for African-Americans. ${ }^{15}$ The association between destinations and physical activity at $1200 \mathrm{~m}$ in our analysis is noteworthy. It may be explained by the fact that, if the association between destination intensity and BMI operates through increased levels of physical activity, then it is likely that stronger associations would be observed at distances such as $1200 \mathrm{~m}$, rather than 400 or $800 \mathrm{~m}$. In order to attain sufficiency in physical activity (and receive the health benefits, such as reduced levels of obesity), people need to be active more often, for longer periods of time. While intensely distributed or clustered destinations at 400 and $800 \mathrm{~m}$ may still encourage physical activity, this may be insufficient to enact effects on BMI, whereas, $1200 \mathrm{~m}$ may be of adequate distance to exert an effect on BMI.

\section{Strengths and limitations}

This present analysis using KDE of destination distribution represents an important advancement in the study of the relationship between the built environment and BMI. KDE expresses the distance and density of destinations. ${ }^{15}$ By using KDE, this study was able to weight or grade destination access, and accommodate the fact that: (1) a set of destinations close to a house is likely to have greater influence on a person than destinations some distance away; and (2) a set of intensely distributed destinations is likely to have greater influence than dispersed destinations.

Another important strength of this research is the way we optimised the specificity of exposure measures by creating exposure areas specific to each individual, rather than creating neighbourhood exposures based on territorially defined area units.

The comprehensive data collection methods (individual surveys, objective environmental audits by trained staff, and the use of publicly available spatial datasets) represent an important strength, and the simultaneous collection of individual and environmental data reduced the risk of bias associated with the misclassification of environmental exposures. 
The use of multiple kernel distances is notable, as it enables the comparison of distance effects, and thereby offers greater ability to observe and understand the complexities of the relationship between destination distribution and BMI. Few previous studies have examined such a wide-ranging list of destinations, particularly in relation to BMI. Of those using KDE to examine the relationship between destinations and BMI, we are not aware of any that have looked beyond food and recreational destinations. While not exhaustive, the wide-ranging list of destinations used here represents an important strength.

There are some limitations that must be acknowledged. First, physical activity and BMI outcome measures are based on self-reported information, and are thus subject to measurement error. ${ }^{36}{ }^{37}$ Comparisons between selfreported and objectively measured BMI show that across the population, height tends to be overestimated and weight underestimated, ${ }^{37} 38$ although this varies by population subgroups. For example, overestimation of height is more common in groups of low socioeconomic status and people with higher $\mathrm{BMI},{ }^{36}$ while weight is more likely to be underestimated by men. ${ }^{39}$ Among women, overestimation of weight has been observed, ${ }^{39}$ however, in the USA, there is some evidence that under-reporting of weight is more prevalent among white, well-educated women. ${ }^{40}$ Self-reported physical activity is also associated with misclassification and systematic error. ${ }^{41}{ }^{42}$ Underestimation of physical activity is more likely for people engaging in high levels of physical activity. ${ }^{42}$ Misclassification of the mediator (in this case physical activity) can severely attenuate estimates of the effects of mediation. Furthermore, as with all such mediation analysis, the model assumes there are no unmeasured confounders, and that there is no misspecification of the causal order. It is also important to acknowledge that $14.2 \%$ of responses were missing for the physical activity variable which may have introduced some bias.

As this is a cross-sectional study, reverse causation is possible. However, we believe that BMI is unlikely to cause destination intensity and that it is more plausible that the direction of effect is from the neighbourhood environment to BMI. It is also true that this analysis is predicated on the assumption that destinations that are more intensely distributed, or clustered, lead to reduced incidence of overweight and obesity. However, it is likely that not all destinations exert healthful effects on BMI; fast-food restaurants, for example, are unlikely to positively improve health. Importantly, however, while it is commonly assumed that the availability of fast-food restaurants is associated with higher BMI, evidence is somewhat mixed: some studies have found positive associations, ${ }^{4} 822$ and others have found no relationship. ${ }^{13}{ }^{43}$ Future analysis of this data set could distinguish between destinations on the basis of their hypothesised relationship with BMI.

It is also important to acknowledge that the participants in this study were adults, so the extent to which the results can be generalised to other populations, such as children and the elderly, is limited. Finally, we have only considered the home environment here. Other environments, such as work and social environments may have important influences on overweight and obesity.

\section{CONCLUSIONS}

This is the first study that we are aware of to assess the relationship between destination intensity and BMI using a wide-ranging set of destinations. We demonstrate that intensely distributed destinations are associated with reduced BMI, most particularly at $1200 \mathrm{~m}$ from home, and that physical activity, at least partly, explains this association. These results have important implications for policy and planning, as they suggest that increasing the density of destinations may lead to reduced levels of obesity by increasing the physical activity of residents.

Acknowledgements The authors thank Gavin Turrell, David Crawford and the late Damien Jolley who were Chief Investigators on this grant, and Emma Rawlings who assisted with the survey administration.

Contributors TLK conceived the paper, conducted the analysis and wrote the paper. AMK, LET, RJB contributed to reviews of the paper.

Funding The VicLANES project was funded by the Victorian Health Promotion Foundation. The first author was supported by a PhD scholarship from the Victorian Health Promotion Foundation.

Competing interests None declared.

Ethics approval Latrobe University Human Ethics Committee.

Provenance and peer review Not commissioned; externally peer reviewed.

Data sharing statement No additional data are available.

Open Access This is an Open Access article distributed in accordance with the Creative Commons Attribution Non Commercial (CC BY-NC 4.0) license, which permits others to distribute, remix, adapt, build upon this work noncommercially, and license their derivative works on different terms, provided the original work is properly cited and the use is non-commercial. See: http:// creativecommons.org/licenses/by-nc/4.0/

\section{REFERENCES}

1. Australian Bureau of Statistics. Australian Health Survey: first results, 2011-2012 (Catalogue no. 4364.0.55.001). Canberra: Australian Bureau of Statistics, 2012.

2. Access Economics. The growing cost of obesity in 2008: three years on. Canberra: Report for Diabetes Australia, 2008.

3. Feng J, Glass TA, Curriero FC, et al. The built environment and obesity: a systematic review of the epidemiologic evidence. Health Place 2010;16:175-90.

4. Dubowitz T, Ghosh-Dastidar M, Eibner C, et al. The Women's Health Initiative: the food environment, neighbourhood socioeconomic status, body mass index and blood pressure. Obesity (Silver Spring) 2012;20:862-71.

5. Black JL, Macinko J, Dixon LB, et al. Neighborhoods and obesity in New York City. Health Place 2010;16:489-99.

6. Bodor JN, Rice JC, Farley TA, et al. The association between obesity and urban food environments. $J$ Urban Health 2010;87:771-81.

7. Wang MC, Kim S, Gonzalez AA, et al. Socioeconomic and food-related physical characteristics of the neighbourhood environment are associated with body mass index. $J$ Epidemiol Community Health 2007;61:491-8.

8. Inagami S, Cohen DA, Brown AF, et al. Body mass index, neighborhood fast food and restaurant concentration, and car ownership. J Urban Health 2009;86:683-95.

9. Thornton LE, Pearce JR, Kavanagh AM. Using geographic information systems (GIS) to assess the role of the built environment in influencing obesity: a glossary. Int $J$ Behav Nutr Phys Act 2011;8:71-9. 
10. Maroko AR, Maantay JA, Sohler NL, et al. The complexities of measuring access to parks and physical activity sites in New York City: a quantitative and qualitative approach. Int $J$ Health Geogr 2009;8:34.

11. Smiley MJ, Diez Roux AV, Brines SJ, et al. A spatial analysis of health-related resources in three diverse metropolitan areas. Health Place 2010;16:885-92.

12. Moore LV, Diez Roux AV, Brines S. Comparing perception-based and geographic information system (GIS)-based characterizations of the local food environment. J Urban Health 2008;85:206-16.

13. Rundle A, Neckerman KM, Freeman L, et al. Neighborhood food environment and walkability predict obesity in New York City. Environ Health Perspect 2009;117:442-7.

14. Kloog I, Haim A, Portnov BA. Using kernel density function as an urban analysis tool: Investigating the association between nightlight exposure and the incidence of breast cancer in Haifa, Israel. Comput Environ Urban Syst 2009;33:55-63.

15. Jones-Smith JC, Karter AJ, Warton EM, et al. Obesity and the food environment: income and ethnicity differences among people with diabetes: the Diabetes Study of Northern California (DISTANCE). Diabetes Care 2013;36:2697-705.

16. King TL, Bentley RJ, Thornton LE, et al. Does the presence and mix of destinations influence walking and physical activity? Int J Behav Nutr Phys Act 2015;12:115.

17. King TL, Thornton LE, Bentley RJ, et al. The use of kernel density estimation to examine associations between neighborhood destination intensity and walking and physical activity. PLOS ONE 2015;10:e0137402.

18. King T, Kavanagh A, Jolley D, et al. Weight and place: a multilevel cross-sectional survey of area-level social disadvantage and overweight/obesity in Australia. Int J Obes 2005;30:281-7.

19. Kavanagh AM, Goller JL, King TL, et al. Urban area disadvantage and physical activity: a multilevel study in Melbourne, Australia. $J$ Epidemiol Community Health 2005;59:934-40.

20. Australian Electoral Commission. Measuring the accuracy of the electoral rolls and testing the effectiveness of continuous roll update a report on the fieldwork from February-March 2004. Canberra: Roll Integrity Unit, Australian Electoral Commission, 2005.

21. Dillman DA. Mail and internet surveys: the Tailored Design Method. 2nd edn. New York: Wiley, 2000.

22. Thornton LE, Bentley RJ, Kavanagh AM. Fast food purchasing and access to fast food restaurants: a multilevel analysis of VicLANES. Int J Behav Nutr Phys Act 2009;6:1-10.

23. Kavanagh AM, Kelly MT, Krnjacki L, et al. Access to alcohol outlets and harmful alcohol consumption: a multi-level study in Melbourne, Australia. Addiction 2011;106:1772-9.

24. ESRI. ArcGIS 10.1. Redlands, CA, 2012

25. Guagliardo MF. Spatial accessibility of primary care: concepts, methods and challenges. Int J Health Geogr 2004;3:3.

26. Aultman-Hall L, Roorda M, Baetz BW. Using GIS for evaluation of neighborhood pedestrian accessibility. J Urban Plann Dev 1997;123:10.

27. Sallis JF, Saelens BE, Frank LD, et al. Neighborhood built environment and income: Examining multiple health outcomes. Soc Sci Med 2009;68:1285-93.
28. Australian Institute of Health and Welfare. The Active Australia survey: A guide and manual for implementation, analysis and reporting. Canberra: AlHW, 2003.

29. Centers for Disease Control and Prevention. Physical activity and health: a report for the Surgeon General. Atlanta, Georgia: US Department of Health and Human Services, 1996.

30. Department of Health. At least five a week: evidence on the impact of physical activity and its relationship to health. London: Department of Health, 2004.

31. Australian Bureau of Statistics. Australian Health Survey: physical activity, 2011-12 (Catalogue No. 4364.0.55.004). Canberra: Australian Bureau of Statistics, 2013.

32. Bauman A, Ford I, Armstrong T. Trends in population levels of reported physical activity in Australia, 1997, 1999 and 2000. Canberra: Australian Sports Commission, 2001.

33. Armstrong T, Bauman A, Davies J. Physical activity patterns of Australian adults. Results of the 1999 National Physical Activity Survey. Canberra: Australian Institute of Health and Welfare, 2000.

34. Rose G. Sick individuals and sick populations. Int J Epidemiol 1985;14:32-8.

35. Rundle A, Diez Roux AV, Freeman L, et al. The urban built environment and obesity in New York City: a mulitlevel analysis. Am J Health Promot 2007;21:326-34.

36. Niedhammer I, Bugel I, Bonenfant S, et al. Validity of self-reported weight and height in the French GAZEL cohort. Int $J$ Obes 2000;24:1111-18.

37. Spencer EA, Appleby PN, Davey GK, et al. Validity of self-reported height and weight in 4808 EPIC-Oxford participants. Public Health Nutr 2002;5:561-5.

38. Connor Gorber SC, Tremblay M, Moher D, et al. A comparison of direct vs. self-report measures for assessing height, weight and body mass index: a systematic review. Obes Rev 2007;8: 307-26.

39. Brug J, Wammes B, Kremers S, et al. Underestimation and overestimation of personal weight status: associations with socio-demographic characteristics and weight maintenance intentions. J Hum Nutr Diet 2006;19:253-62.

40. Craig BM, Adams AK. Accuracy of body mass index categories based on self-reported height and weight among women in the United States. Matern Child Health J2009; 13:489-96.

41. Mahabir S, Baer DJ, Giffen C, et al. Comparison of energy expenditure estimates from 4 physical activity questionnaires with doubly labeled water estimates in postmenopausal women. $A m \mathrm{~J}$ Clin Nutr 2006;84:230-6.

42. Maddison R, Ni Mhurchu $\mathrm{C}$, Jiang $\mathrm{Y}$, et al. International Physical Activity Questionnaire (IPAQ) and New Zealand Physical Activity Questionnaire (NZPAQ): a doubly labelled water validation. Int $J$ Behav Nutr Phys Act 2007;4:62.

43. Mobley LR, Root ED, Finkelstein EA, et al. Environment, obesity, and cardiovascular disease risk in low-income women. Am J Prev Med 2006;30:327-332. 